\title{
Location preference and flavor aversion reinforced by amphetamine in rats
}

\author{
MURRAY A. REICHER and ERIC W. HOLMAN \\ University of California, Los Angeles, California 90024
}

\begin{abstract}
To associate the identical drug state with both a location and a flavor, rats were given intraperitoneal amphetamine injections and then confined for $20 \mathrm{~min}$ in one side of a shuttlebox with access to a flavored solution; on control trials without injections, they were confined for $20 \mathrm{~min}$ in the opposite side with a different flavor. In the first experiment, the rats were placed in the shuttlebox immediately after injections; in the second experiment, they were placed in the shuttlebox $20 \mathrm{~min}$ after injections. Subsequent free-choice tests in both experiments revealed an increased choice of the side of the shuttlebox associated with amphetamine but also an aversion to the flavored solution associated with the drug.
\end{abstract}

In attempts to characterize the reinforcing properties of abused drugs, a paradox has arisen. Cappell and Le Blanc (1973) found aversive taste conditioning by intraperitoneal amphetamine within the range of doses which Pickens and Harris (1968) found rats would selfinject intravenously. Thus, a single pharmacological stimulus may act as both a positive and a negative reinforcer depending on the associated stimuli and responses.

Wise, Yokel, and deWit (1976) pointed out, however, that these seemingly paradoxical effects could be explained by differences in procedural variables such as route of drug administration, extent of drug habituation, and delay of reinforcement. Wise et al. therefore attempted to control for these factors in demonstrating positive and negative reinforcement by intravenous amphetamine and apomorphine. Their first experiment circumvented habituation difficulties by giving rats that had previously shown amphetamine self-administration a 10-min exposure to saccharin followed by an amphetamine injection. A subsequent test indicated moderate suppression of saccharin drinking. However, as the report acknowledges, this does "not . . . establish that a particular drug injection can be both reinforcing and aversive" (p. 1273). The difficulty is that positive reinforcement was demonstrated by self-administered amphetamine immediately following leverpressing, while negative reinforcement was demonstrated by a single, experimenter-administered amphetamine injection given only after a 10-min drinking period. In the second experiment by Wise et al., rats previously trained to selfadminister amphetamine were given access to saccharin

This research was supported by UCLA Academic Senate Research Committee Grant 2386. We would like to thank Gaylord D. Ellison and R. A. Wise for helpful comments and suggestions. Reprints may be obtained from Eric W. Holman, Department of Psychology, University of California, Los Angeles, California 90024. followed immediately by an apomorphine self-injection session. Some of the rats continued to leverpress for apomorphine, yet subsequently showed an aversion to the saccharin. As Wise et al. mentioned, however, there were still difficulties in this procedure, including the difference in the delay of reinforcement for leverpressing and saccharin-drinking, as well as the possible ambiguity created by first training the rats to self-administer amphetamine and then suddenly switching to apomorphine.

The present experiments avoided some of these problems by associating the same injections with a compound stimulus consisting of a distinctive flavor and a distinctive location; thus, positive location preferences and taste aversions were established simultaneously.

\section{EXPERIMENT 1}

\section{Method}

Subjects. The subjects were 12 iemale albino rats, 120-150 days old and weighing about $210 \mathrm{~g}$, bred from Sprague-Dawley stock in the UCLA Psychology Department colony.

Apparatus. The rats were trained in a rectangular $(70 \times 30 \times$ $25 \mathrm{~cm}$ ) wooden shuttlebox with a grid floor and a transparent Plexiglas roof. During training periods, a thin wooden barrier was placed in the shuttlebox which restricted the rats to the appropriate side only. The walls of the right side were unfinished wood, while the walls of the left side were cross-hatched with $1-\mathrm{cm}$-wide black tape. The shuttlebox was also placed at an oblique angle to the ceiling light in the room. Therefore, the subjects' cues presumably included both the interior of the shuttlebox itself and a differential light and shadow pattern. There were two drinking solutions: the banana solution contained .19\% sodium saccharin and $2 \%$ Crown Colony banana extract in water, and the almond solution contained $.19 \%$ sodium saccharin and $2 \%$ Crown Colony almond extract in water. They were provided to the rats in graduated test tubes with drinking spouts. There was a small hole in each end of the shuttlebox, through which the drinking spout was placed, allowing the rats access to an appropriately flavored solution during training. The amphetamine injections $\left(1.43^{\circ} \mathrm{mg} / \mathrm{kg}\right)$ were administered intraperitoneally in a volume of about $2 \mathrm{ml}$.

Procedure. The rats lived in individual cages with constant 
temperature and illumination and had access to unlimited water. They were handled daily beginning 10 days before the experiment and were fed $12 \mathrm{~g}$ per day of Purina Lab Chow shortly after training or handling. During the experiment, the rats were run individually once daily, with both training and test periods lasting $20 \mathrm{~min}$. The experimental schedule included 12 days of training and 1 test day, followed by 8 days of retraining and 2 days for the final tests. On even-numbered training days, each rat was removed from its home cage, given an amphetamine injection and immediately placed in one side of the shuttlebox with access to a distinctly flavored solution, as described earlier. On odd-numbered days, each rat received no amphetamine and was placed in the opposite side of the shuttlebox with access to a differently flavored solution. Half the rats were placed in the left side of the shuttlebox on drug days and half in the right; half the rats in each group received the banana flavored solution in the left side and half received it in the right side. Thus, there were four balanced subgroups containing three subjects each.

Independent tests were performed to determine the rats' acquired side and flavor preferences. For the first tests, on Day 13, no amphetamine was administered. Each rat was placed in the middle of the shuttlebox with access to both sides, but no solutions were provided; the rat's location was automatically recorded for $20 \mathrm{~min}$. Immediately after the side-preference test, the rat was returned to its cage and offered the banana solution and the almond solution in test tubes side by side for $20 \mathrm{~min}$; total consumption of each solution was measured. The final tests, on Days 22 and 23, repeated this procedure, except that the flavor preference test occurred 1 day after the side preference test instead of immediately after it, and each subject received an amphetamine injection immediately before both tests.

\section{Results}

Table 1 presents the mean daily consumption of each flavor by each group during the 20 days of training. Every subject drank less on amphetamine days than on control days ( $p<.001$ by a sign test), indicating the anorexic effect of the drug.

Table 2 presents the mean consumption of each flavor by each group in the tests. Subjects showed an aversion to the flavor associated with amphetamine: in the no-drug test, 11 of 12 subjects avoided the amphetamine flavor and 1 prefererred it ( $p<.01$ by a sign test), and in the drug test, 8 subjects avoided the amphetamine flavor and 4 were tied $(\mathrm{p}<.05$ by a sign test). The anorexic effect of amphetamine was again reflected in a lower total consumption by each rat $(\mathrm{p}<.001$ by a sign test) in the drug test than in the no-drug test.

Table 3 presents the mean percent of the 20 -min test period that each group of subjects stayed on the left side during the tests. The groups that had received amphetamine on the left spent more time on the left in each test than did the groups that had received amphetamine on the right. This effect of amphetamine reinforcement

Table 1

Mean Daily Consumption (ml) of Almond/Banana Flavors in Training for Each Group

\begin{tabular}{cccc}
\hline \multirow{2}{*}{$\begin{array}{c}\text { Flavor Associated } \\
\text { With Amphetamine }\end{array}$} & \multicolumn{3}{c}{ Side Associated With Amphetamine } \\
\cline { 2 - 4 } & Left & Right & Mean \\
\hline Almond & $1.0 / 9.0$ & $1.0 / 7.6$ & $1.0 / 8.3$ \\
Banana & $4.5 / 1.1$ & $8.7 / 1.0$ & $6.6 / 1.0$ \\
\hline
\end{tabular}

Table 2

Mean Consumption (ml) of Almond/Banana Flavors in Tests for Each Group

\begin{tabular}{cccc} 
Flavor Associated & \multicolumn{3}{c}{ Side Associated With Amphetamine } \\
\cline { 2 - 4 } With Amphetamine & \multicolumn{1}{c}{ Left } & Right & Mean \\
\hline & \multicolumn{3}{c}{ No-Drug Test } \\
Almond & $0.7 / 7.0$ & $0.0 / 5.0$ & $0.3 / 6.0$ \\
Banana & $14.7 / 0.7$ & $8.0 / 1.3$ & $11.3 / 1.0$ \\
& & Drug Test & \\
Almond & $0.3 / 1.0$ & $0.3 / 0.7$ & $0.3 / 0.8$ \\
Banana & $2.0 / 0.2$ & $1.3 / 0.3$ & $1.7 / 0.2$ \\
\hline
\end{tabular}

Table 3

Mean Percent Choice of Left Side in Tests for Each Group

\begin{tabular}{ccc} 
Flavor Associated & \multicolumn{3}{c}{ Side Associated With Amphetamine } \\
\cline { 2 - 3 } With Amphetamine & Left & Right \\
\hline & \multicolumn{3}{c}{ No-Drug Test } \\
Almond & 67.9 & 58.2 \\
Banana & 65.1 & 38.9 \\
Mean & 66.5 & 48.5 \\
& & Drug Test \\
Almond & 82.0 & 61.7 \\
Banana & 64.6 & 51.8 \\
Mean & 73.3 & 56.7 \\
\hline
\end{tabular}

was significant according to a three-way mixed-design analysis of variance $[F(1 / 8)=36.7, p<.001]$, and did not differ between the drug and no-drug tests $[F(1 / 8)$ $=.1]$. The effect was also significant in each test separately according to two-way analyses of variance $[F(1 / 8)$ $=45.4, \mathrm{p}<.001$, for the no-drug test, and $F(1 / 8)=$ $11.2, \mathrm{p}<.025$, for the drug test]. At the level of individual subjects averaged over both tests, all but one of the rats that received amphetamine on the left spent more time on the left than did all but one of the rats that received amphetamine on the right.

Other significant, but less interesting, effects in the three-way analysis were that the rats spent more time on the left during the drug test than during the no-drug test $[F(1 / 8)=7.4, p<.05]$, and the rats that had received amphetamine with the almond flavor spent more time on the left than did the rats that had received amphetamine with the banana flavor $[\mathrm{F}(1 / 8)=18.7, \mathrm{p}<.005]$. No other main effects or interactions were significant. Side preferences were tabulated in 2-min blocks and showed no consistent trends during either test.

\section{Discussion}

The data indicate that amphetamine positively reinforced a side preference in the shuttlebox yet produced a flavor aversion; the learned preference and aversion were expressed by both drugged and nondrugged subjects. These results, however, do not conclusively differentiate whether the positive and negative reinforcing effects of amphetamine are temporally simultaneous or 
successive. Since location cues are associated mainly with immediate reinforcement while flavor cues can be associated with more delayed reinforcement (Garcia, Ervin, \& Koelling, 1966), the present results could conceivably occur if the amphetamine injection were positively reinforcing at first and negatively reinforcing later. To evaluate this possibility, the experiment was repeated with a slightly modified procedure designed to associate both flavor and location with relatively delayed effects of amphetamine.

\section{EXPERIMENT 2}

\section{Method}

The subjects were 12 female albino rats, $120-150$ days old, and weighing about $210 \mathrm{~g}$, bred from Sprague-Dawley stock at the UCLA Biology Department colony. Materials and methods were identical to those used in the first experiment. However, the procedure included the following modification: On training and test days, the subjects remained in their individual cages after amphetamine administration for a 20 -min period before being placed in the shuttlebox, instead of being placed in the shuttlebox immediately after amphetamine administration.

\section{Results}

Table 4 presents the mean daily consumption of each flavor during the 20 days of training. As in the first experiment, every subject drank less on amphetamine days than on control days ( $p<.001$ by a sign test), indicating the anorexic effect of the drug.

Table 5 presents the consumption of each flavor in the tests. In the no-drug test, subjects showed an aversion to the flavor associated with amphetamine: 10 of 12 subjects avoided the amphetamine flavor, 1 preferred it, and 1 was tied $(\mathrm{p}<.05$ by a sign test). In the drug test, however, subjects demonstrated no significant flavor preference: 3 subjects avoided the amphetamine flavor, 3 preferred it, and 6 were tied. The anorexic effect of amphetamine was once again reflected in a lower total consumption by each rat $(p<.001$ by a sign test) in the drug than in the no-drug test.

Table 6 presents the mean percent of the 20 -min test period that each group of subjects spent on the left side during the tests. The groups that had received amphetamine on the left spent more time on the left in each test than did the groups that had received amphetamine on the right. This effect of amphetamine reinforcement was significant according to a three-way mixed-design analysis of variance $[F(1 / 8)=29.2, p<$ $.001]$, and was significantly greater in the drug test than in the no-drug test $[F(1 / 8)=37.1, p<.001]$. The effect was also significant in each test separately according to two-way analyses of variance $[F(1 / 8)=13.4, p<.01$, for the no-drug test, and $F(1 / 8)=16.5, p<.01$, for the drug test]. Averaged over both tests, all the rats with amphetamine on the left spent more time on the left than did all the rats with amphetamine on the right. No other main effects or interactions were significant. Side preferences were tabulated in 20-min blocks and showed no consistent trends during either test.

\section{Discussion}

The data from the second experiment indicate that even with a 20 -min delay between administration and training, amphetamine positively reinforced a side preference in the shuttlebox yet produced a flavor aversion; the learned preference was expressed in both drugged and nondrugged subjects, whereas the aversion was expressed only in nondrugged subjects. If amphetamine is positively reinforcing at first and negatively reinforcing later, then the second experiment, in which the cues were associated with a later part of the drug effect, should have shown a weaker location preference and a stronger flavor aversion than the first experiment. The data showed no such differences in the no-drug tests, and, if anything, opposite effects in the drug tests. It therefore seems more reasonable to conclude that amphetamine can be simultaneously positively and negatively reinforcing, depending on the cue. Since location cues are more easily associated with exteroceptive reinforcers while flavor cues are more easily associated with interoceptive reinforcers (Garcia \& Koelling, 1966),

Table 4

Mean Daily Consumption (ml) of Almond/Banana Flavors in Training for Each Group

\begin{tabular}{cccc} 
Flavor Associated & \multicolumn{3}{c}{ Side Associated With Amphetamine } \\
\cline { 2 - 4 } With Amphetamine & \multicolumn{1}{c}{ Left } & Right & Mean \\
\hline Almond & $1.8 / 7.4$ & $1.7 / 7.7$ & $1.7 / 7.5$ \\
Banana & $12.9 / 2.1$ & $9.7 / 1.6$ & $11.3 / 1.8$ \\
\hline
\end{tabular}

Table 5

Mean Consumption ( $\mathrm{ml}$ ) of Almond/Banana Flavors in Tests for Each Group

\begin{tabular}{|c|c|c|c|}
\hline \multirow{2}{*}{$\begin{array}{l}\text { Flavor Associated } \\
\text { With Amphetamine }\end{array}$} & \multicolumn{3}{|c|}{ Side Associated With Amphetamine } \\
\hline & Left & Right & Mean \\
\hline & \multicolumn{3}{|c|}{ No-Drug Test } \\
\hline Almond & $7.3 / 6.7$ & $3.3 / 5.7$ & $5.3 / 6.2$ \\
\hline \multirow[t]{2}{*}{ Banana } & $19.0 / 3.3$ & $11.7 / 1.0$ & $15.3 / 2.1$ \\
\hline & \multicolumn{3}{|c|}{ Drug Test } \\
\hline Almond & $1.7 / 1.7$ & $0.7 / 0.3$ & $1.2 / 1.0$ \\
\hline Banana & $0.7 / 1.0$ & $1.3 / 0.5$ & $1.0 / 0.7$ \\
\hline
\end{tabular}

Table 6

Mean Percent Choice of Left Side in Tests for Each Group

\begin{tabular}{ccc} 
Flavor Associated & \multicolumn{3}{c}{ Side Associated With Amphetamine } \\
\cline { 2 - 3 } With Amphetamine & \multicolumn{3}{c}{ Left } & No-Drug Test \\
\hline & \multicolumn{3}{c}{ Right } \\
Almond & 66.4 & 48.4 \\
Banana & 69.6 & 57.6 \\
Mean & 68.0 & 53.0 \\
& & Drug Test \\
Almond & 73.4 & 39.6 \\
Banana & 85.0 & 43.3 \\
Mean & 79.2 & 41.5 \\
\hline
\end{tabular}


amphetamine may have a positive effect on exteroceptive stimuli while having a negative interoceptive effect.

In conclusion, the present results confirm and extend the findings of Wise et al. (1976), with intraperitoneal rather than intravenous amphetamine as the reinforcer and side preference rather than leverpressing as the positively reinforced response. The present experiment may offer a relatively convenient method for testing the reinforcing properties of other drugs as well.

\section{REFERENCES}

Cappell, H., \& Le Blanc, A. E. Punishment of saccharin drinking by amphetamine in rats and its reversal by chlor- diazepoxide. Joumal of Comparative and Physiological Psychology, 1973, 85, 97.104.

Garcia, J., Ervin, F. R., \& Koelling, R. A. Learning with prolonged delay of reinforcement. Psychonomic Science, 1966 , 5, 121-122.

Garcia, J., \& Koelling, R. A. The relation of cue to consequence in avoidance learning. Psychonomic Science, 1966. 4, 123-124.

Pickens, R., \& Harris, W. C. Self-administration of d-amphetamine by rats. Psychopharmacologia, 1968, 12. 158-163.

WISE, R. A., Yokel, R. A., \& DEWIT, H. Both positive reinforcement and conditioned aversion from amphetamine and from apomorphine in rats. Science, 1976, 191, 1273-1274.

(Received for publication May 2, 1977; accepted May 20, 1977.) 